\title{
Cost-effective optoelectronic system to measure the projectile velocity in high-velocity impact testing of aircraft and spacecraft structural elements
}

\author{
José M. Sánchez-Pena, MEMBER SPIE \\ Carlos Marcos \\ María Y. Fernández \\ Universidad Carlos III de Madrid-E.P.S. \\ Departamento de Tecnología Electrónica \\ Grupo de Displays y Aplicaciones Fotónicas \\ Avda de la Universidad, 30 \\ E28911, Leganes, Madrid, Spain \\ E-mail: jmpena@ing.uc3m.es
}

\author{
Ramón Zaera \\ Universidad Carlos III de Madrid-E.P.S. \\ Departamento de Mecánica de Medios \\ Continuos y Teoría de Estructuras \\ Avda de la Universidad, 30 \\ E28911, Leganés, Madrid, Spain
}

\begin{abstract}
We present a microcontrolled optoelectronic system to measure online the average velocity of a projectile impacting on aircraft and spacecraft structures. The projectile velocity can vary in the range from subsonic to supersonic. The implemented optical system is based on three optical barriers that are crossed by the projectiles before impacting on the structural elements. A simple optoelectronic prototype is constructed and tested. The flight times among the three optical barriers are obtained by the microcontroller system. The measured velocity accuracy in test conditions is better than $1 \%$. A high-brightness liquid crystal display is used to show messages of the system configuration and also the measured projectile velocity. To ensure safety in the experimental impact tests, serial RS-232 communication can be used to monitor this information using a remote computer. The system is highly robust since it is able to measure projectile velocity even when an optical barrier is missed by failure in either the optical transmitter or the receptor. () 2007 Society of Photo-Optical Instrumentation Engineers. [DOI: 10.1117/1.2740771]
\end{abstract}

Subject terms: optical barriers; microcontroller system; flight time; high-velocity impact testing; aircraft structures.

Paper 060765SSR received Sep. 29, 2006; revised manuscript received Nov. 30, 2006; accepted for publication Dec. 15, 2006; published online May 18, 2007.

\section{Introduction}

Aircraft structures may be subjected to a variety of environmental impact hazards. Radome, radar antenna, landing lights, canopy, windshield, lateral section or intake of the engine nacelle, turbine blades, wing or tail empennage leading edges, and fuel tanks inside wings are eventually targets of high-velocity impact loading during flight, takeoff, and landing. Today, the risk of structural and system failures, as well as of occupant injuries, is well recognized in aircraft design. Different structural parts of airplanes and helicopters are currently designed to deflect the impacting body trajectory and to partially absorb its impact energy. ${ }^{1}$

There are a number of different causes for these shocks. Bird strikes $^{1}$ and hailstones ${ }^{2}$ are the most critical because of their high probability of occurrence and their disastrous consequences-especially when ingested by the engine. Also the ice released from the edge of a propeller blade may impact the nacelle of the twin engine or the fuselage, and a rubber tire fragment may impact the underside of wing structures. ${ }^{3}$ The Concorde accident in 2000 was actually caused by a tire fragment impacting a fuel tank on the wing of the airplane. Such an impact results in a pressure wave that propagates through the fluid within the tank, stresses the walls, and increases the risk of catastrophic wing failure. Another catastrophic example was the impact of frozen lightweight foam projectiles on the wing of the Space Shuttle. Other kinds of projectiles that may impact

0091-3286/2007/\$25.00 @ 2007 SPIE aircraft structures are small and medium caliber bullets and fragments due to a blast, both of them causing hydrodynamic ram effects when impacting the fuel tank of an airplane or a helicopter. The aeroengine turbine blade may also fail due to fatigue and may penetrate the wall of the containment cell, damaging oil tanks and airframes. ${ }^{4}$

Impact engineering is also of great interest in the field of spacecraft. The near-earth space environment, where satellites, the International Space Station, and the Space Shuttle orbit the earth, is jumbled with both artificial and natural debris. These debris are too small and numerous to be individually tracked and there are many satellites in orbit, so that that the average time between destructive collisions is about 10 years.

A great effort was made recently to design advanced shielding to protect aircraft and spacecraft structures and to evaluate the damage on structures impacted by projectiles and meteoroids. Although numerical simulation has been increasingly adopted to analyze these problems, experimental testing is still necessary to validate numerical codes and obtain reliable information. Among the variables that should be measured during an impact test, an accurate determination of the velocity of the projectile is obviously of great importance, because of its quadratic relation to the kinetic energy applied to the specimen. The most common measurement system is the chronograph. The velocity of the projectile is calculated by dividing the base length, or the distance between two sensor locations, by the difference of the times when the projectile arrives at these locations. The type of sensor used to detect the passage of a solid 
distinguishes different chronographs. Among these sensors, the simplest are the break-paper and the break-wire sensors. In the first type, two sheets of aluminum, separated by a sheet of paper, are connected to a circuit. The projectile triggers a signal when it impacts the laminate of sheets. Nevertheless, this system leads commonly to a small number of faults, especially when the projectile is nonmetallic. In the second type, which is more reliable than the first, a thin wire is cut by the projectile. The main drawback of both systems is related to impacts at subsonic velocities: the pressure wave preceding the projectile causes stretching before breaking of the wire or bulging before tearing of the sheets. The base length then varies, and this leads to an unknown error.

Inductive sensors are more sophisticated and offer better accuracy. The system operation is as follows. A circular open-coil sensor is located surrounding the path of the projectile. When the projectile approaches, eddy currents are induced and the voltage output increases, reaching a peak and then decreasing as the bullet leaves the coil. The system must be calibrated and the gain setting must be adjusted for each projectile material, size, and shape by manually positioning it in the coil before the test. In addition, those projectiles made of nonconductive material can not be detected by this type of sensor.

Another measurement system is Doppler radar, also called continuous-wave radar. An antenna is aligned to the projectile path and sends a continuous radio signal. The projectile reflects the pulses with a frequency shifted due to the Doppler effect, enabling it to produce a target frequency after being mixed with a sample of the transmit frequency. The Doppler radar method and apparatus for measuring a projectile's muzzle velocity offers important benefits: good accuracy, simple setup, and minimal risk of equipment damage by stray bullets. An additional advantage is the possibility to measure, in some cases, the residual velocity of the projectile after it perforates the target. Nevertheless, a conventional system is quite expensive compared to chronographs.

Photographic systems have been also used to determine the velocity of a projectile. Since the event takes place quickly and time exposures should be extremely short (of the order of a microsecond or even shorter), ultra-highspeed cameras must be used. In these, the high-speed film usually remains stationary inside a drum and sweeps the image across it by reflection from a rotating mirror or prism. ${ }^{5,6}$ Facing film-based cameras, digital ultra-highspeed cameras passed through a striking development in the last decade. The appearance of highly light-sensitive megapixel CMOS sensors enabled the acquisition of images with an exposure time short enough to freeze the movement of the projectile. The easy-to-operate approach of these cameras and the possibility to record for long time periods facilitates the synchronization and triggering operations. In any case, the high cost of analog or digital cameras - and sometimes that of the lighting systems necessary to illumine the projectile-currently constitutes a significant drawback.

Optical sensors seem to be the most commonly used devices to trigger the counter. The opposite of a screen of photo-optic sensors, an array of bulbs provides a light curtain that is perturbed by the passage of the projectile. This

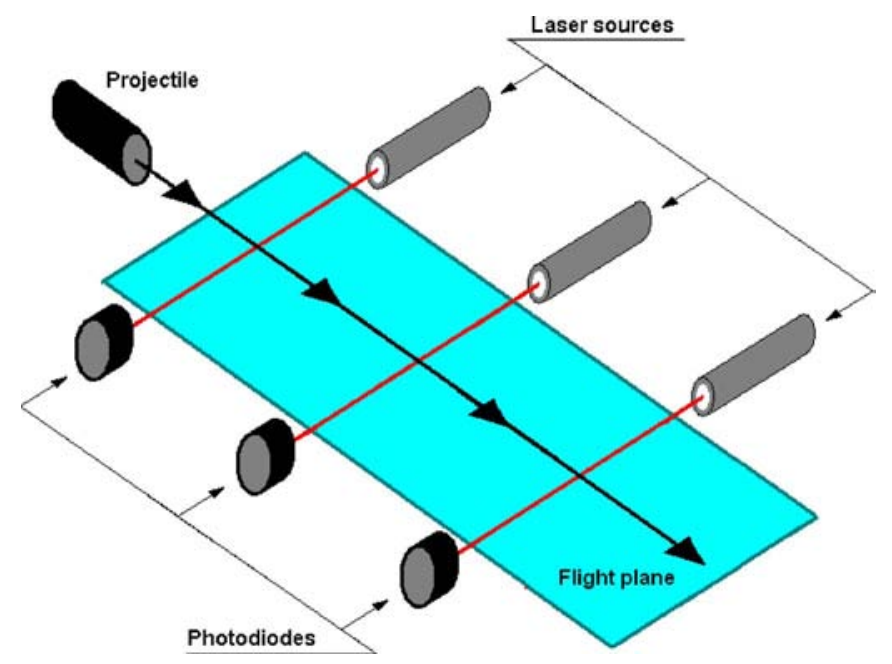

Fig. 1 Optical configuration of velocity-measuring system.

causes a fluctuation in the light intensity level received by the screen. When outdoor tests are performed, the lightbulbs could be obviated so that the light from the sky above the screen is modified when the projectile passes immediately overhead. IR LEDs can also be used to illuminate the photodetector. Optical sensors are not expensive, but the detection task is not always trivial, especially when an array of bulbs or LEDs is used. In such cases, small-caliber pellets may fill only a small part of the sensor, and a preliminary test to adjust the light and the gain of the screen must be performed before any conclusive experiment. Then a laser beam opposed to a photodiode can easily solve this difficulty since the beam is completely screened by the projectile if the sensor is suitably positioned along the path of the projectile.

In this paper, we present a simple, cost-effective, and robust microcontrolled optoelectronic system that is able to measure with a reasonable accuracy a wide range of projectile velocities for impact testing of aeronautic and aerospace structures.

\section{System Design}

The optical system is based on three equidistant optical barriers, as shown in Fig. 1. Each barrier consists of a beam laser and a photosensor. The whole system uses three laser sources and three photodiodes to form three light lines. Each light line is combined with its own signal-processing circuit, which is managed by the microcontroller. The three optical barriers are on the same level forming a plane. A projectile can be measured only when traveling through this flight plane, therefore the railgun must be positioned perpendicular to the laser barriers.

The projectile velocity is measured through the acquisition of three different signals coming from the crossing of the optical barriers by the projectile during its flight. The detection of these signals by the photosensors and their processing by the microcontroller enables the system to evaluate the average velocity of the projectile. The working principle is as follows. The photodiodes sense when lasers are blocked by the projectile trajectory that is supposed to be perpendicularly incident to the sequential laser barriers. 


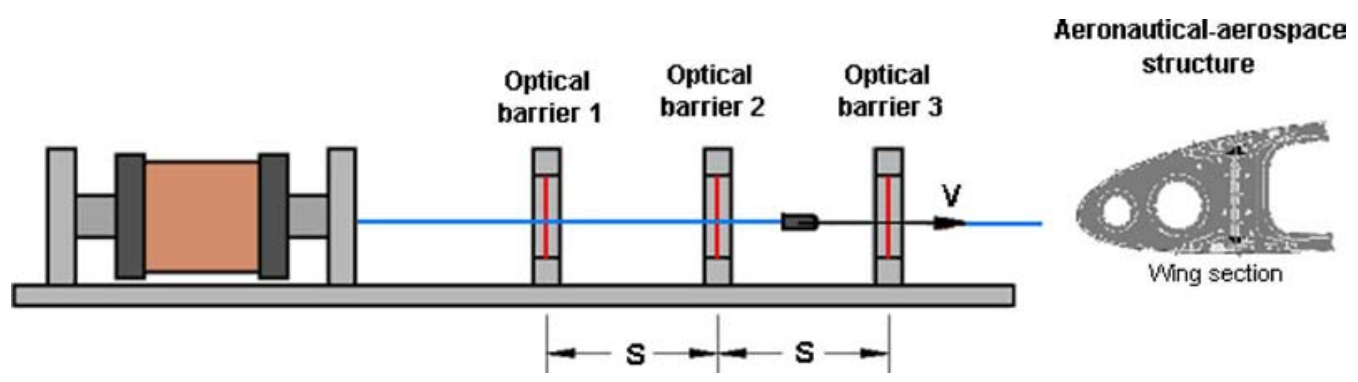

Fig. 2 System schematic showing the optical barriers, the projectile, and the test structure.

When the projectile crosses the first optical barrier, the laser beam is blocked and a microcontroller time counter is triggered. Flight times are registered when second and third laser beams are also crossed by the projectile, and then three flight times between laser barriers are determined (Fig. 2).

If $S$ is the distance between laser barriers that are equidistant, then the average speed $v$ for the projectile is given by

$v=1 / 3\left(\frac{S}{T_{1}}+\frac{S}{T_{2}}+\frac{2 S}{T_{3}}\right)$,

where $T_{1}$ is the flight time for the projectile between the first and second optical barriers, $T_{2}$ is between the second and third barriers, and finally, $T_{3}$ is the flight time between first and third barriers. Supposedly, $T_{3}$ must be the sum of $T_{1}$ plus $T_{2}$, but it could be different because the system is counting microcontroller clock periods. Therefore, projectile velocity is calculated as an average, using three distances between barriers and three flight times. This simple method is robust against potential failures of either the light sources or the photodetectors.

On the other hand, the deceleration suffered by the projectile due to aerodynamic drag could be estimated by the following equation

$m_{p} \frac{\mathrm{d} v}{\mathrm{~d} t}=-\frac{1}{2} A_{p} C_{D}(v) \rho_{a} v^{2}$

where $v$ is the velocity of the projectile, $m_{p}$ is its mass, $A_{p}$ is its cross-sectional area, $C_{D}$ is the drag coefficient, and $\rho_{a}$ is the density of the air. After a straightforward calculation, this equation leads to

$$
\frac{\mathrm{d} v}{\beta(v) v}=-\mathrm{d} x,
$$

where $\beta$ is a function of the velocity. Typical values of $\beta$ vary from $5 \times 10^{-4}$ to $5 \times 10^{-2}$ in ballistic applications, considering low-density (polymers) or high-density (tungsten, uranium) projectiles; different shapes (blunt, spherical, conical, or ogival noses), cross-sectional areas; and velocities at subsonic, transonic, or fully supersonic ranges. Assuming a small variation of velocity when the projectile passes through the system, that must be confirmed afterward, we can consider $C_{D}$-and $\beta$ - as constant. The variation of velocity $\Delta v$ relative to the initial velocity $v_{0}$ as a function of the distance traveled by the projectile $x$ could then be determined by direct integration of Eq. (3):

$\frac{\Delta v}{v_{0}}=\exp (-\beta x)-1$.

As can be observed in Fig. 3, the variation of velocity between the first and the last laser barrier of the system, obtained with Eq. (4), is equal to $1 \%$ in the worst situation (projectile of small density, small radius, and high aerodynamic drag).

\section{System Configuration and Working Principle}

The designed system includes an electro-optical block, a sensor-signal-processing unit, an LCD display for monitoring the information, a timing count unit, and a central processing unit (Fig. 4).

The electro-optical block consists of three 1-mW output power and 635-nm laser diodes directed onto high-speed silicon photodiodes. These components form the optical barriers. Additionally, this block also includes the necessary hardware to check the right operation of the optical barriers, and in case of failure, the central processing unit is informed and necessary actions are taken by the microcontroller to keep the system operative.

Figure 5 shows the circuits integrating the sensorprocessing unit. Its main function is to generate the trigger signal required by the timing count unit. Additionally, this block is also responsible to evaluate residual environmental

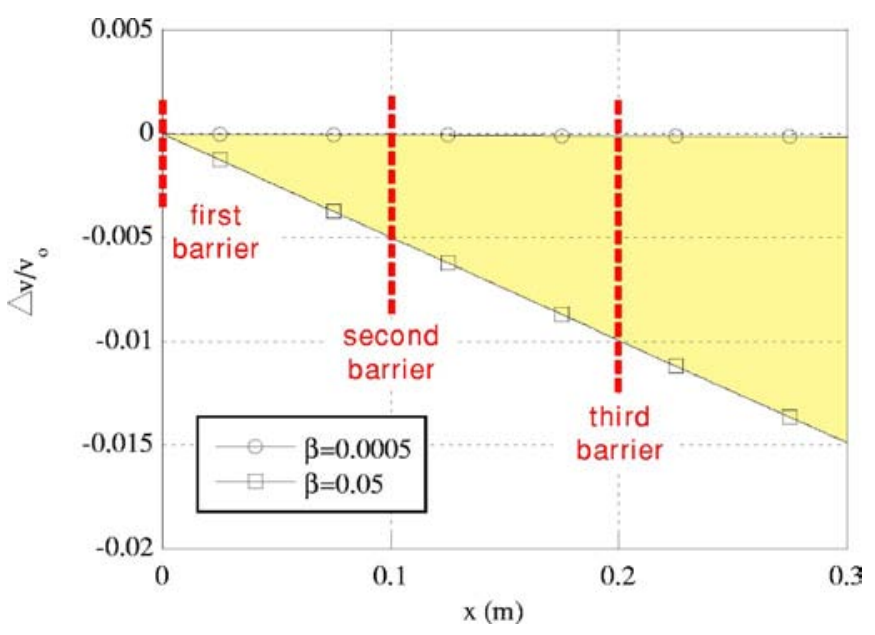

Fig. 3 Variation of velocity $\Delta v$ relative to the initial velocity $v_{0}$ as a function of the distance traveled by the projectile $x$. 


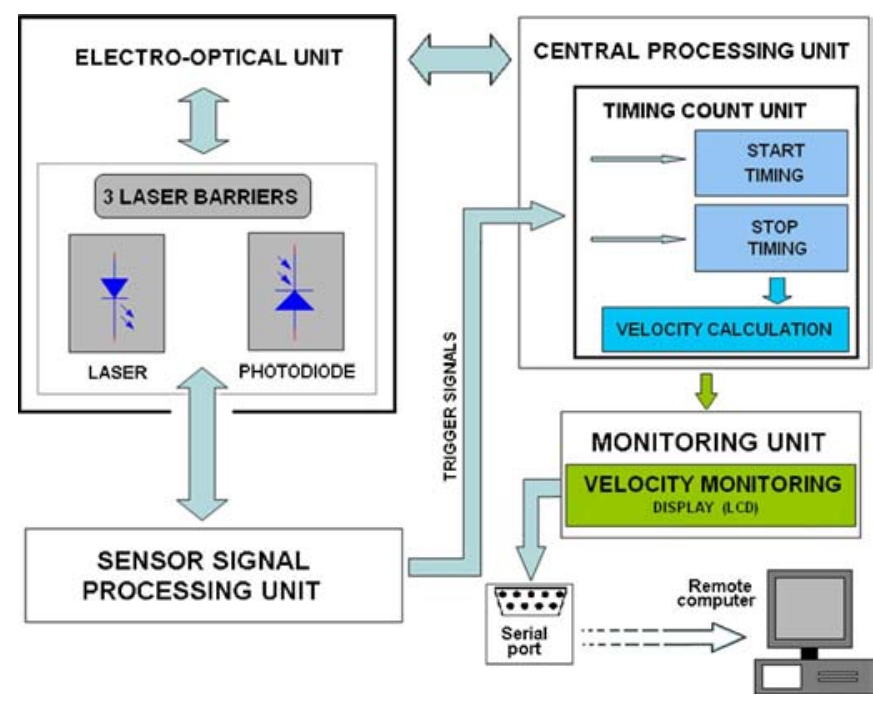

Fig. 4 Block diagram of the whole system.

lighting. Thus, the system can set a voltage comparator level in the sensor circuit and compare it to photodiode sensor voltage, knowing that laser beams have been blocked by the projectile when the comparator state changes. A customized circuit was implemented to provide the voltage level in the photodiode sensors with the lasers on and off. This circuit is based on a pulse width modulation (PWM) generator and a low-pass filter. PWM is a powerful technique that is employed in a wide variety of applications, ranging from measurement and communications, to power control and conversion. The output signals of the customized circuit coming from the low-pass filter provide a continuous voltage level that depends on the PWM duty cycle. The continuous voltage level at the output filter is compared to the photodiode circuit voltage when the lasers are off, registering the PWM duty cycle when the comparator level changes. The same process is followed when the lasers switch from off to on states, registering the new duty cycle value. To ensure that the sensor-signal-processing unit generates the appropriate trigger signal when the laser beam is blocked, the mean value of the registered PWM signal is calculated so that the PWM generated will have the previously obtained duty cycle.

The display unit (LCD) shows different information related to the configuration parameters of the system and the

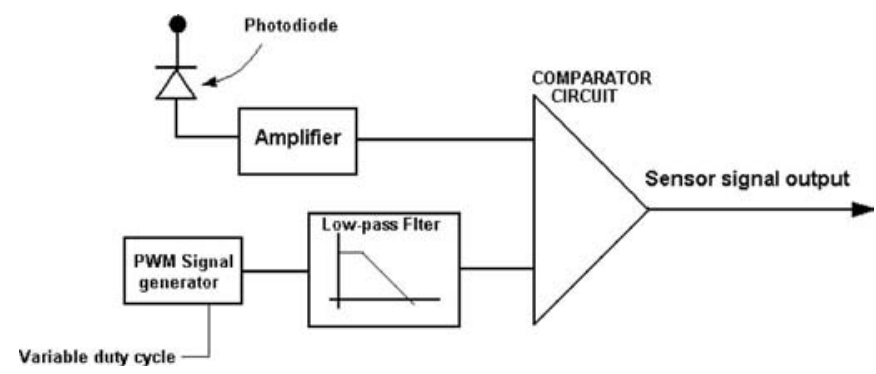

Fig. 5 Sensor-signal-processing unit.

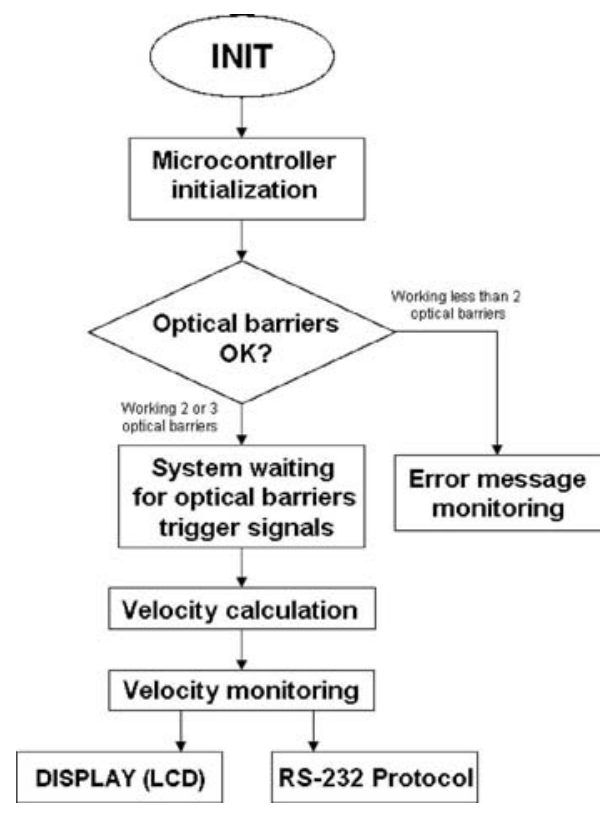

Fig. 6 Flowchart of the system performance.

measured projectile velocity. To ensure safety in laboratory tests, the information can also be sent via a serial bus and collected by a remote computer.

The main control and processing unit (CPU) was implemented with the AT89C2051 Atmel microcontroller based on the 8051 architecture, which is compatible with the industry-standard MCS-51 instruction set and provides a highly flexible and cost-effective solution to many embedded control applications.

Figure 6 shows a simplified flowchart used by the CPU to implement its basic functions. It must supervise the rest of the system units and properly manage trigger signals from the sensor unit. Once the system is configured, the control and processing unit waits for a trigger signal coming from the sensor-signal-processing unit. This trigger signal is generated once a projectile passes through the optical barriers. Then the timing count unit registers the flight times and it is able to calculate the average projectile velocity, sending the measurement to the monitoring unit (LCD).

Unlike other sophisticated systems that use light grids ${ }^{7}$ to build the target area points or beam expanders to form a light screen, ${ }^{8}$ our implemented system presents advantages such as low cost due to the reduced number of electrical and optical elements used, a high robustness against potential failures of the optical sources or detectors, simple operation, and a reasonable accuracy in the measurement.

\section{Results and Discussion}

The evaluation of the measurement accuracy in this kind of metrology system is very important since it is related to the square of the kinetic energy applied to the tested aeronautic/aerospace structures. To avoid potential damage of such structural elements in real working operation, this parameter should be precisely evaluated to obtain the right conclusions in high-velocity impact testing performed on such elements. 


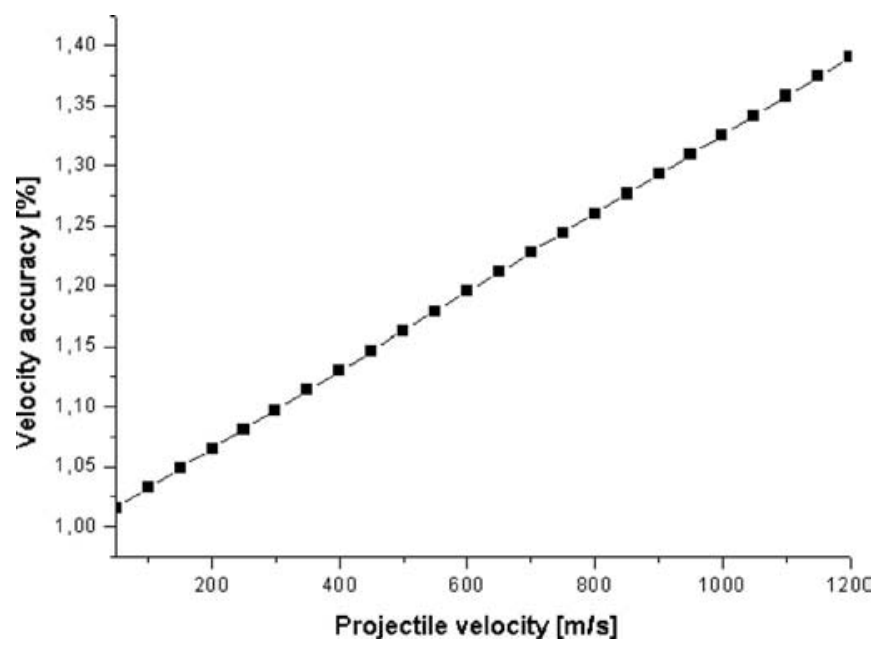

Fig. 7 Theoretical velocity accuracy of the system working in the worst case with only two optical barriers.

In our proposed system and in the worst case, when only two optical barriers are working, the accuracy of the projectile velocity measurement was deduced as

$$
\left|\frac{\Delta v}{v}\right| \leqslant \frac{|\Delta S|+v|\Delta T|}{S},
$$

where the parameters are the same as used in Eq. (1). Here $\Delta v, \Delta S$, and $\Delta T$ are values of maximum error in $v, S$, and $T$, respectively.

The absolute accuracy of the proposed system is limited by at least two factors: (1) the accuracy with which the optical barriers are set out and (2) the microcontroller time required to acquire trigger signals from the optical barriers. In our case, the distance between optical barriers is $10 \mathrm{~cm} \pm 1 \mathrm{~mm}$ (positioning error), and the microcontroller response time is usually 6 times the microcontroller clock cycle $\left(32.5 \times 10^{-8} \mathrm{~s}\right)$.
Several parameters may limit the measurement accuracy in optical metrology systems. 9,10 Some of them can be avoid or minimized using more complex processing circuits for the signals coming from the optical sensors or using appropriate algorithms in experimental data processing. Figure 7 presents accuracy as a function of the projectile velocity when the system is working in the worst case, i.e., only two optical barriers are operative.

A customized test circuit was implemented to evaluate the proposed system and to check the accuracy. The test circuit is necessary because the movement of an object is difficult to perform by mechanical methods. The implemented test circuit is based on a microcontroller circuit that can control the laser switching, making it possible to simulate a projectile crossing the laser barriers. To do that, a dwell time is programmed to generate the switching off sequence of the lasers. Twelve velocities were simulated from 100 to $1200 \mathrm{~m} / \mathrm{s}$, and we acquired 20 measurements for each. The accuracy experimentally obtained is shown in Fig. 8. Using this circuit it is possible to simulate a wide range of projectile velocities without doing expensive real tests (shoots). As we can see in Fig. 8, a slight deviation of the measured velocity compared to the "ideal" velocity appears along the whole range of measurement. In all cases, the experimental value for the projectile velocity is lower than the expected value. This biased deviation may be attributed to a systematic error in the process of acquiring and processing the experimental data. However, its influence is not relevant for the specific tests considered here.

The measuring system was checked under normal operation conditions (using the three optical barriers), and it provided an accuracy much better than in the previous case, where only two optical barriers were considered (Fig. 9).

\section{Conclusions}

An optoelectronic system to measure projectile velocity online was designed and constructed with discrete circuits. The system is based on the measurement of projectile flight times between three optical barriers. Velocity data are cal-

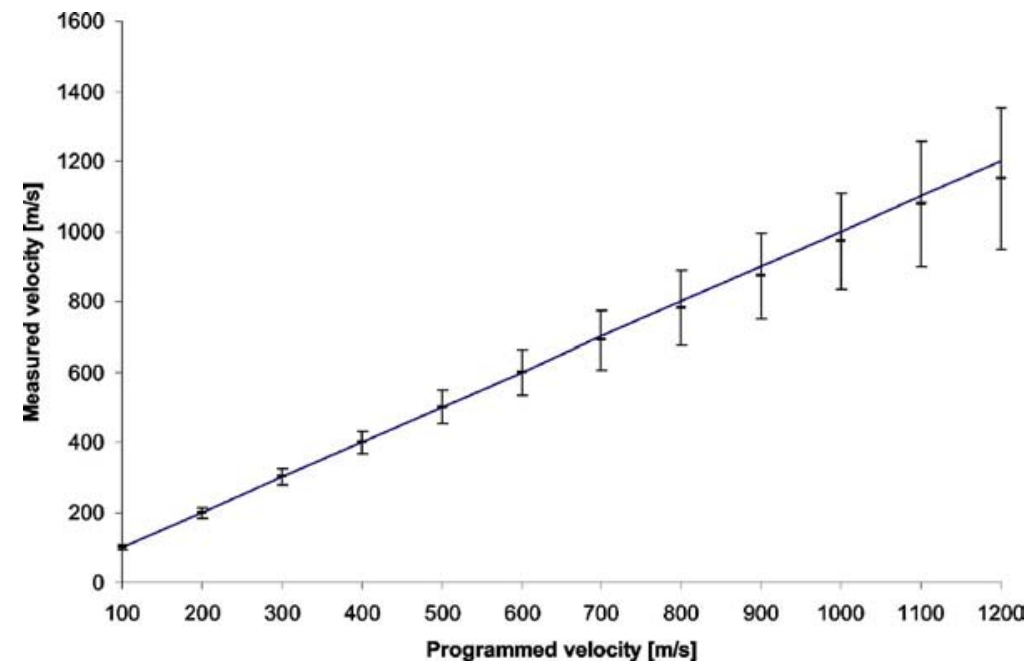

Fig. 8 Velocity measured by the proposed optoelectronic system versus programmed velocity. The error bars are not to scale, and the solid line represents the "ideal" velocity that should be obtained in the measurement. 


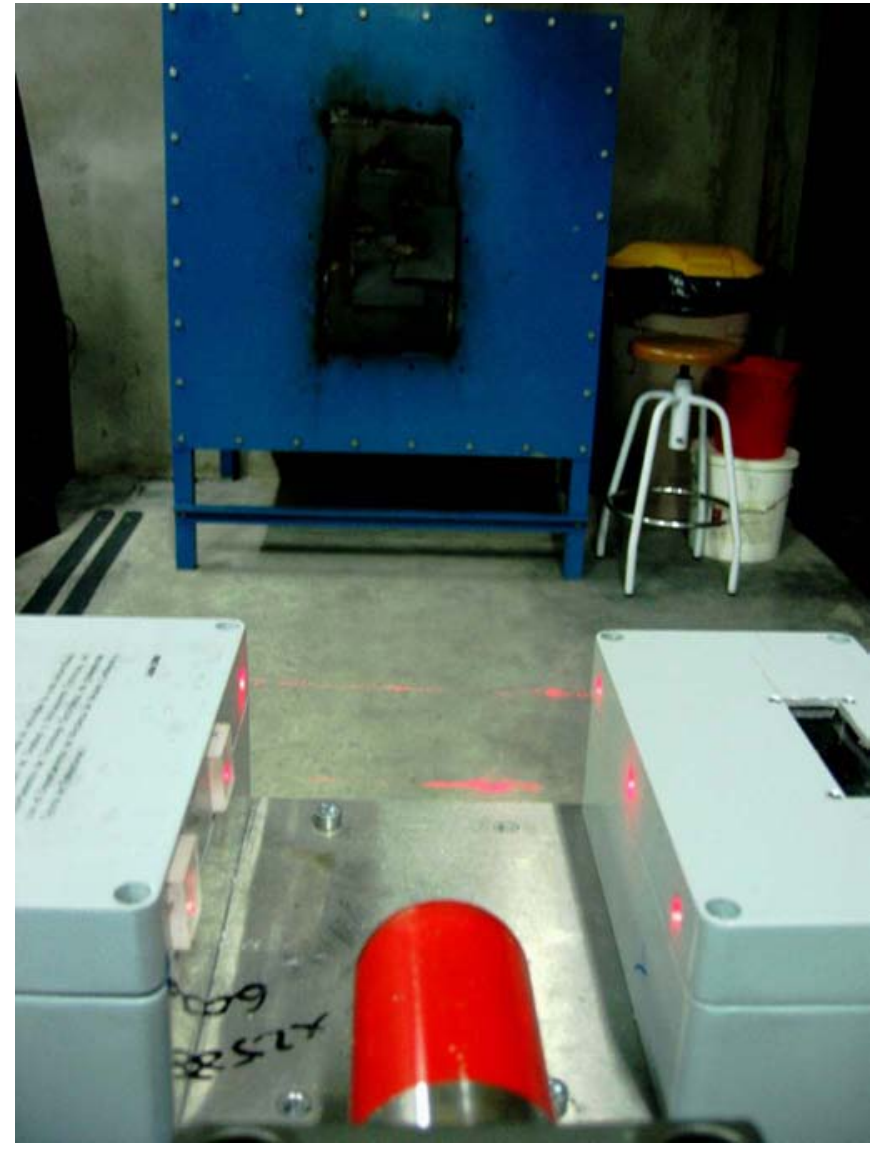

Fig. 9 Photograph of the real system used for high-velocity impact testing of aircraft and spacecraft structures.

culated by the control process unit (microcontroller) and displayed on an LCD mounted in the system or on a remote computer using a serial communication. The velocity accuracy was theoretically calculated and experimentally evaluated. Values less than $1 \%$ were obtained for the worst case. This accuracy depends mainly on the projectile velocity and optical barrier distances, and it could be improved by increasing either the clock frequency of microcontroller or the distance between optical barriers. The influence of background light in the measured velocity is negligible. The implemented system is simple, cost-effective, and robust against potential failures of the optical elements and covers a wide velocity range from subsonic to supersonic.

\section{ACKNOWLEDGMENT}

This work was partially supported by CAM (FACTOTEMCM: S-0505/ESP/000417).

\section{References}

1. A. Airoldi and B. Cacchione, "Modelling of impact forces and pressures in Lagrangian bird strike analyses," Int. J. Impact Eng. 32, 1651-1677 (2006).

2. M. Anghileri, L. M. L. Castelletti, F. Invernizzi, and M. Mascheroni, "A survey of numerical models for hail impact analysis using explicit finite element codes," Int. J. Impact Eng. 31, 929-944 (2005).

3. R. A. W. Mines, S. McKown, and R. S. Birch, "Impact of aircraft rubber tyre fragments on aluminium alloy plates: I-Experimental," Int. J. Impact Eng. 34, 627-646 (2007).

4. X. Hai-jun and W. Rong-ren, "Aeroengine turbine blade containment tests using high-speed rotor spin test facility," Aerosp. Sci. Technol.
10, 501-508 (2006)

5. J. A. Zukas, T. Nicholas, H. F. Swift, L. B. Greszczuk, and D. R. Curran, Impact Dynamics, Chapter 7, Ch. Krieger Publishing, FL (1992).

6. G. M. Moss, D. W. Leeming, and C. L. Farrar, Military Ballistics, Chap. 7, Brassey's New Battlefield Weapons Systems and Technology Series into the 21st Century, London (1995).

7. E. C. Crittenden, R. A. King, and T. C. Andrews, "Target measurement system for precise projectile location," U.S. patent No. 3727069 (1973).

8. L. L. Deck, "An optical device for rapid measurement of the speed, dispersion, attack angle and shock wave of high velocity small caliber projectiles," in Proc. 10th Int. Symp. on Ballistics, Vol. 1, pp. 1-9 (1987).

9. R. Hartwig, "Accuracy of velocity measurement of projectiles with fins and tracers by means of sky-screens," J. Ballist. 9(3), 2299-2310 (1986).

10. K. A. Schroder, R. J. Allen, J. V. Parker, and P. T. Snowden, "In-bore measurements using an optical data link," IEEE Trans. Magn. 35(1), 95-99 (1999).

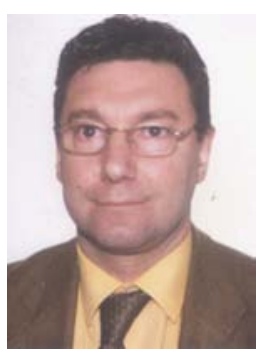

José M. Sánchez-Pena received his MS and $\mathrm{PhD}$ degrees in telecommunication engineering from Polytechnic University of Madrid in 1988 and 1993, respectively. From February to September 1993, he was a visiting researcher at Exeter University, United Kingdom, engaged in modeling the electro-optical properties of liquid crystal displays (LCDs). In March 1995, he joined Carlos III University of Madrid, where he is currently an associate professor and heads the Displays and Photonic Applications Group in the Electronic Technology Department. His main research interests include electro-optical characterization of LCDs, optical sensors, and optoelectronic instrumentation for rehabilitation technology applications and optical communications. He is a member of SPIE and ILCS.

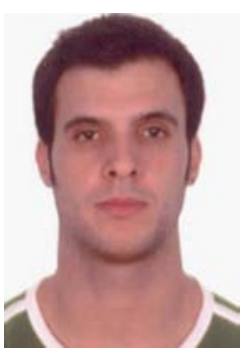

Carlos Marcos received his MS degree in industrial engineering in 2006 from Carlos III University of Madrid, where he is an assistant professor with the Electronic Technology Department. He is currently working toward a doctoral degree on electro-optical optimization and addressing of optical LED (OLED) microdisplays.

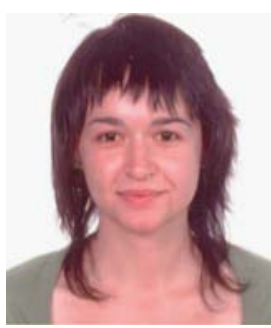

María Y. Fernández received her BS degree in electronic engineering in 2006 from Carlos III University of Madrid. She is currently, working toward her MS degree in advanced electronic systems.

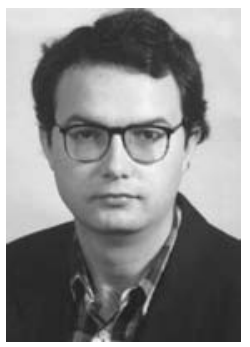

Ramón Zaera is an associate professor with the Department of Continuum Mechanics and Structural Analysis of Carlos III University of Madrid. He received his BS degree in civil engineering and $\mathrm{PhD}$ degree in civil engineering, in 1997, both from Polytechnic University of Madrid. His current research interests are numerical and analytical simulation of ballistic impact problems, dynamic characterization of materials, crashworthiness and dynamic instabilities. 